\title{
Heat flow into spheres for a class of energies
}

\author{
Norbert Hungerbühler \\ Department of Mathematics, University of Fribourg, Pérolles, 1700 Fribourg SwitzerLAND
}

\begin{abstract}
Let $M$ and $N$ be compact smooth Riemannian manifolds without boundaries. Then, for a map $u: M \rightarrow N$, we consider a class of energies which includes the popular Dirichlet energy and the more general $p$-energy. Geometric or physical questions motivate to investigate the critical points of such an energy or the corresponding heat flow problem. In the case of the Dirichlet energy, the heat flow problem has been intensively studied and is well understood by now. However, it has turned out that the case of the $p$-energy $(p \neq 2)$ is much more difficult in many respects. We give a survey of the known results for the $p$-harmonic flow and indicate how these results can be extended to a larger class of energy types by using Young measure techniques which have recently been developed for quasilinear problems.
\end{abstract}

\section{Introduction}

\section{$1.1 \quad$ The $p$-energy}

Let $M$ and $N$ be smooth Riemannian manifolds without boundary of dimension $m$ and $n$ respectively. $M$ and $N$ are equipped with Riemannian metrics $\gamma$ and $g$. The $p$-energy of a differentiable map $u: M \rightarrow N$ is defined to be

$$
E_{p}(u):=\frac{1}{p} \int_{M}|D u|^{p} d \mu .
$$

Here, $D u: T_{x} M \rightarrow T_{u(x)} N$ is the differential of $u$ to which we associate the $p$-energy density

$$
e_{p}(u):=\frac{1}{p}|D u|^{p}=\frac{1}{p}\left(\operatorname{trace}\left((D u)^{*} D u\right)\right)^{p / 2},
$$

where ${ }^{(*)}$ denotes the adjoint with respect to the underlying inner product on the respective tangent spaces $T_{x} M$ and $T_{u(x)} N$. And, of course, $d \mu$ is the measure on $M$ associated with the metric on $M$. In local coordinates, the $p$-energy can be expressed by the following formula:

$$
E_{p}(u):=\frac{1}{p} \int_{M}\left(\gamma^{\alpha \beta}\left(g_{i j} \circ u\right) \partial_{\alpha} u^{i} \partial_{\beta} u^{j}\right)^{p / 2} \sqrt{\gamma} d x
$$

Here, $\sqrt{\gamma}=\sqrt{\left|\operatorname{det}\left(\gamma_{\alpha \beta}\right)\right|}$, and we adopt the usual summation convention, i.e. we automatically sum over Latin indices from 1 to $n$ and over Greek indices from 1 to $m$. By Nash's embedding theorem, we always may assume that $N$ is isometrically embedded in some Euclidean space $\mathbb{R}^{k}$. In that case, the $p$-energy density is given by

$$
\frac{1}{p}\left(\gamma^{\alpha \beta} \partial_{\alpha} u \cdot \partial_{\beta} u\right)^{p / 2}
$$

where $u: M \rightarrow N \subset \mathbb{R}^{k}$ is viewed as a map into $\mathbb{R}^{k}$ and '.' is the Euclidean inner product in $\mathbb{R}^{k}$.

We remark, that for $p=2$ the $p$-energy coincides with the well known Dirichlet energy. Moreover, as it is easy to see, the $p$-energy is conformally invariant iff $m=p$. This fact allows to switch between conformal charts. 


\subsection{The first variation of the $p$-energy}

Given a class $F$ of mappings from $M$ to $N$ defined by boundary conditions on $\partial M$ (if there is a boundary) and possibly additional topological conditions, e.g. a homotopy class, one may try to minimize the $p$-energy within the class $F$. One may hope that the solution of this minimization problem is a map $u$ in $F$ with particular analytic and geometric properties. In physics, the $p$-energy for maps into the sphere or into a Grassmannian manifold may serve as a model for liquid crystals. Stationary points of the energy then correspond to physical equilibria of the liquid.

Necessarily, in a minimum $u$, the first variation of the $p$-energy must vanish at $u$ for all variations of $u$ with compact support in the interior of $M$. Let us consider variations $u_{t}: M \rightarrow N$ of $u=u_{0}$ for $|t|$ small such that $u_{t}(x)$ is of class $C^{1}$ in the variables $(x, t)$ and $u_{t}(x)=u(x)$ for all $t$ and all $x$ outside some compact subset $K \subset M$ which is contained in the domain $U$ of a coordinate system $x^{1}, \ldots, x^{m}$ on $M$ with range $\Omega$ and is mapped into the domain of a fixed coordinate system $y^{1}, \ldots, y^{m}$ on $N$ by all $u_{t}$. Now, the support of the initial vector field of the variation

$$
V(x):=\left.\frac{d}{d t} u_{t}(x)\right|_{t=0} \in T_{u(x)} N
$$

is contained in $K$. We also assume that $V$ is of the class $C^{1}$. It is no restriction to assume $u_{t}^{i}(x)=u^{i}(x)+t V^{i}(x)$. If $u$ is $C^{2}$, the usual computation yields that vanishing first variation is equivalent to

$$
\begin{aligned}
\frac{1}{\sqrt{\gamma}} \frac{\partial}{\partial x^{\beta}}\left(\sqrt{\gamma}\left(\gamma^{\alpha \beta} g_{i j} \frac{\partial u^{i}}{\partial x^{\alpha}} \frac{\partial u^{j}}{\partial x^{\beta}}\right)^{\frac{p}{2}-1} \gamma^{\alpha \beta} \frac{\partial u^{l}}{\partial x^{\alpha}}\right)= \\
=-\left(\gamma^{\alpha \beta} g_{i j} \frac{\partial u^{i}}{\partial x^{\alpha}} \frac{\partial u^{j}}{\partial x^{\beta}}\right)^{\frac{p}{2}-1} \gamma^{\alpha \beta} \Gamma_{i j}^{l} \frac{\partial u^{i}}{\partial x^{\alpha}} \frac{\partial u^{j}}{\partial x^{\beta}}
\end{aligned}
$$

in $U$ for $l=1, \ldots, n$. Here, we used the Christoffel symbols

$$
\Gamma_{i j}^{l}=\frac{1}{2} g^{l k}\left(g_{i k, j}-g_{i j, k}+g_{j k, i}\right)
$$

of the metric $g$ with respect to the coordinates chosen on $N$. Thus, the right hand side can be interpreted as

$$
A(u)(D u, D u)\left(p e_{p}(u)\right)^{1-2 / p}
$$

where $A(u)(\cdot, \cdot)$ denotes the second fundamental form of $N$. The operator on the left hand side of (1) is called $p$-Laplace operator related to the manifolds $M$ and $N$ and is denoted by $\Delta_{p}$, i.e. $\Delta_{2}$ is simply the Laplace-Beltrami operator of the manifold $M$ and does not depend on $N . \Delta_{2}$ is a linear elliptic diagonal operator in divergence form. For $p>2$ $(0<p<2)$ the operator is degenerate (singular) at points $D u=0$.

The right hand side of (1) is for $p=2$ a quadratic form in the first derivatives of $u$ with coefficients depending on $u$. These strong nonlinearities are caused by the non-Euclidean structure of the target manifold $N$ and cannot be removed by special choices of coordinates on $N$ unless $N$ is locally isometric to Euclidean space $\mathbb{R}^{n}$. But even in this case the space of mappings from $M$ to $N$ does not possess a natural linear structure unless $N$ itself is a linear space. In general, the right hand side of (1) is of the order of the $p$-th power of the gradient of $u$. 
A $C^{2}$-solution $u: M \rightarrow N$ of (1) is called $p$-harmonic map.

Using variations in the domain, i.e. variations of the form

$$
u_{t}(x)=u(x+t \zeta(x))
$$

where $\zeta=\left(\zeta^{1}, \ldots, \zeta^{m}\right)$ with each $\zeta^{j} \in C_{0}^{\infty}\left(B_{\rho}(y)\right)$, we obtain from $\left.\frac{d}{d t} u_{t}(x)\right|_{t=0}=0$ an equation whose classical solutions contain the set of the $p$-harmonic maps. However its weak solutions, which are called "stationary $p$-harmonic maps", need not contain the set of weak solutions of (1). In particular, $p$-energy minimizing maps are both, weakly and stationary $p$-harmonic. Weakly $p$-harmonic maps admit in general far worse singularities than the energy minimizing maps. Many results are known about stationary $p$-harmonic maps. See e.g. Duzaar and Fuchs [9], Fuchs [18] and [19], as well as Strzelecki [41] and [40].

If we think of $N$ as being isometrically embedded in some $\mathbb{R}^{k}$, we consider the following variations: Let $S \subset \mathbb{R}^{k}$ be a tubular neighborhood of $N$ and $\pi_{N}: S \rightarrow N$ the (smooth) nearest-neighbor projection. Denote $T_{p} N \subset T_{p} \mathbb{R}^{k}$ the tangent space to $N$ at a point $p \in N$. Let $\varphi \in C_{0}^{1}\left(M, \mathbb{R}^{k}\right)$ satisfy

$$
\varphi(x) \in T_{u(x)} N
$$

for all $x \in M$ and $\varphi$ having compact support in a single coordinate chart of $M$. Then $\varphi$ induces a $C^{1}$-variation at $u: M \rightarrow N \subset \mathbb{R}^{k}$ :

$$
u_{t}=\pi_{N} \circ(u+t \varphi) \text {. }
$$

Now, the first variation of the $p$-energy is given by

$$
\begin{aligned}
\left.\frac{d}{d t} E_{p}\left(u_{t}\right)\right|_{t=0} & =\int_{M}\left(\gamma^{\alpha \beta} \frac{\partial u}{\partial x^{\alpha}} \cdot \frac{\partial u}{\partial x^{\beta}}\right)^{\frac{p}{2}-1} \gamma^{\alpha \beta} \frac{\partial u}{\partial x^{\alpha}} \cdot \frac{\partial \varphi}{\partial x^{\beta}} \sqrt{\gamma} d x= \\
& =-\int_{M} \Delta_{p} u \cdot \varphi \sqrt{\gamma} d x
\end{aligned}
$$

where $\Delta_{p}$ is the $p$-Laplace operator related to $M$ and $\mathbb{R}^{k}$, i.e.

$$
\Delta_{p} u=\frac{1}{\sqrt{\gamma}} \frac{\partial}{\partial x^{\beta}}\left(\sqrt{\gamma}\left(\gamma^{\alpha \beta} \frac{\partial u^{j}}{\partial x^{\alpha}} \frac{\partial u^{j}}{\partial x^{\beta}}\right)^{\frac{p}{2}-1} \gamma^{\alpha \beta} \frac{\partial u}{\partial x^{\alpha}}\right) .
$$

Thus, the first variation vanishes at a $C^{2}$ map $u$ if and only if

$$
\Delta_{p} u \perp T_{u} N .
$$

We can make (2) more explicit by introducing a local orthonormal frame $\nu_{n+1}, \ldots, \nu_{k}$ for $\left(T_{p} N\right)^{\perp}$, the orthogonal complement of $T_{p} N$ in $\mathbb{R}^{k}$. Then, by (2) there exist scalar functions $\lambda^{n+1}, \ldots, \lambda^{k}$ such that

$$
-\Delta_{p} u=\sum_{l=n+1}^{k} \lambda^{l}\left(\nu_{l} \circ u\right) .
$$

Multiplying (3) by $\nu_{i} \circ u\left(i\right.$ fixed) and using the fact that $\frac{\partial u}{\partial x^{\alpha}} \cdot \nu_{l}(u)=0$ for all $\alpha$, we obtain

$$
\lambda^{i}=\left(\gamma^{\alpha \beta} \frac{\partial u^{j}}{\partial x^{\alpha}} \frac{\partial u^{j}}{\partial x^{\beta}}\right)^{\frac{p}{2}-1} \gamma^{\alpha \beta} \frac{\partial u^{j}}{\partial x^{\alpha}} \frac{\partial \nu_{i}^{j}(u)}{\partial x^{\beta}}
$$


The Euler-Lagrange equations of the $p$-energy can also be formulated in the following coordinate free form:

$$
e(u) \tau(u)+\left(1-\frac{2}{p}\right) D u \operatorname{grad}_{\gamma} e(u)=0 .
$$

Here, $\tau(u)$ denotes the tension field of $u: \tau(u)=\operatorname{trace}_{\gamma} \nabla D u$, and $\nabla$ denotes the pull-back covariant derivative in the bundle $T^{*} M \otimes u^{-1} T N$. For $p=2$ the equation reduces simply to

$$
\tau(u)=0 .
$$

As an application, we see, that the identity mapping $\operatorname{id}_{M}: M \rightarrow M$ of a Riemannian manifold is $p$-harmonic: Since $D\left(\operatorname{id}_{M}\right)$ has constant coefficients with respect to all coordinate systems on $M, \nabla D\left(\operatorname{id}_{M}\right)=0$ and the first term in (5) vanishes. On the other hand, the $p$-energy density $e\left(\mathrm{id}_{M}\right)$ is constant and hence the gradient in the second term disappears, too. We remark that in general $\mathrm{id}_{M}$ is not $p$-energy minimizing within its homotopy class (see Eells-Lemaire [11]).

The natural space to work in when considering questions concerning the $p$-energy is the nonlinear Sobolev space

$$
W^{1, p}(M, N):=\left\{f \in W^{1, p}\left(M, \mathbb{R}^{k}\right) ; f(x) \in N \text { for } \mu \text {-almost all } x \in M\right\} .
$$

Notice, that if both $M$ and $N$ are compact, then different embeddings of $N$ in $\mathbb{R}^{k}$ give rise to homeomorphic spaces $W^{1, p}(M, N)$. For an intrinsic definition of the space $W^{1, p}(M, N)$ see Federer [17]. If $N$ is isometrically embedded in $\mathbb{R}^{k}$, then the formulas defining the $p$-energy for $C^{1}$ functions make sense also for functions in $W^{1, p}(M, N)$. If a function $u \in$ $W^{1, p}(M, N)$ is a weak solution of the Euler-Lagrange equations we discussed previously, then it is called a weakly $p$-harmonic map.

The space $H^{1, p}(M, N)$ defined as the closure of the class of smooth functions from $M$ to $N$ in the $W^{1, p}$-norm is contained in $W^{1, p}(M, N)$ but does not coincide with the latter space in general (this fact gives rise to the so called "gap phenomenon" of Hardt-Lin [21]). This important observation was first made by Schoen and Uhlenbeck: see Eells and Lemaire [11] as a main reference. However, we have $H^{1, p}(M, N)=W^{1, p}(M, N)$ if $\operatorname{dim}(M)=p$ (see Schoen and Uhlenbeck [39], Bethuel [2] or Bethuel and Zheng [3]). The gap phenomenon limits the possibilities to approximate the $p$-harmonic flow, which we will discuss next, by solutions with smooth initial data.

\subsection{The $p$-harmonic flow}

A basic existence problem for $p$-harmonic maps is the homotopy problem:

Given a map $u_{0}: M \rightarrow N$ is there a p-harmonic map u homotopic to $u_{0}$ ?

This question was first answered in the case $p=2$ of harmonic maps. The answer is affirmative if the sectional curvature $K^{N}$ of $N$ is non-positive (see Eells-Sampson [12]), or-in case of a two-dimensional surface $M$-if the second fundamental group of $N$ is trivial: $\pi_{2}(N)=0$ (see Lemaire [30] and Sacks-Uhlenbeck [38]). Eells and Wood destroyed the hope for a more general theorem by the following counterexample in [13] by showing that, if $u: T^{2} \rightarrow S^{2}$ is 2-harmonic, then $\operatorname{deg} u \neq \pm 1$. Another counterexample has been given by Lemaire in [30]. Thus, in general the attempt to solve the homotopy problem by minimizing $E$ within a given homotopy class will fail: Homotopy classes are not weakly 
closed in $W^{1, p}(M, N)$ generally. Thus, it may be difficult or impossible to solve the homotopy problem for $p$-harmonic maps by direct variational methods. As the key idea to get around this difficulty, Eells and Sampson proposed in [12] to study the heat flow related to the 2-energy:

$$
u_{t}-\Delta_{2} u=A(u)(\nabla u, \nabla u)_{M} \quad \text { on } M \times[0, \infty[
$$

with initial and boundary data

$$
u=u_{0} \text { at } t=0 \text { and on } \partial M \times[0, \infty[
$$

for maps $u: M \times\left[0, \infty\left[\rightarrow N \subset \mathbb{R}^{k}\right.\right.$. Here, $A(u): T_{u} N \times T_{u} N \rightarrow\left(T_{u} N\right)^{\perp}$ is the second fundamental form of $N$. The idea behind this strategy is of course that a continuous deformation $u(\cdot, t)$ of $u_{0}$ will remain within the given homotopy class. Since (6) may be interpreted as the $L^{2}$-gradient flow for the 2-energy, one may hope that the solution $u(\cdot, t)$ for $t \rightarrow \infty$ will come to a rest at some critical point of $E_{2}$ that is a harmonic map. For target manifold $N$, satisfying the geometric restrictions mentioned above, this program has been applied with success for $p=2$.

Another approach to the homotopy problem for $p$-harmonic maps has been given by Duzaar and Fuchs in [10]: they extended the Eells-Sampson result to the case $p \in[2, \infty)$ by using an asymptotic analysis of the not degenerate energy $\int_{M}\left(\varepsilon+|D u|^{2}\right)^{p / 2} d \mu, \varepsilon>0$.

Corresponding to the harmonic flow, the $p$-harmonic flow is described by

$$
u_{t}-\Delta_{p} u=(p e(u))^{1-\frac{2}{p}} A(u)(D u, D u) \quad \text { on } M \times[0, \infty[
$$

with initial data

$$
u=u_{0} \text { at } t=0
$$

for maps $u: M \times\left[0, \infty\left[\rightarrow N \subset \mathbb{R}^{k}\right.\right.$.

We now want to briefly describe the known results concerning the $p$-harmonic flow. We restrict this presentation to the case $p \neq 2$.

The $p$-harmonic flow was first considered in [6] and [22]. There, the following result has been proved for the $p$-harmonic flow into spheres, i.e. for

$$
\begin{aligned}
\partial_{t} u-\Delta_{p} u & =p e_{p}(u) u & & \text { on } M \times[0, \infty[ \\
u(\cdot, 0) & =u_{0} & & \text { on } M \\
|u| & =1 & & \mu \text {-a.e. on } M \times[0, \infty[.
\end{aligned}
$$

Theorem 1 For initial data $u_{0} \in W^{1, p}\left(M, S^{n}\right), p \geq 2$, there exists a global weak solution $u$ to the equation (8)-(10). This solution is weakly continuous in $t>0$ with values in $W^{1, p}(M)$, i.e. for any test function $g \in C^{\infty}(M), h_{1}(t)=\int_{M} u \cdot g d \mu$ and $h_{2}(t)=$ $\int_{M} D u \cdot D g d \mu$ are in $C^{0, \frac{1}{2}}\left(\mathbb{R}_{+}\right)$. Furthermore for almost every $t \geq 0$ this solution satisfies the energy inequality

$$
\int_{0}^{t}\left\|\partial_{t} u\right\|_{L^{2}(M)}^{2} d t+E_{p}(u(t)) \leq E_{p}\left(u_{0}\right)
$$

The technique was the same as in the corresponding case of the harmonic flow which was solved by Chen in [5]: The idea is a penalization technique to approximate the $p$-harmonic 
flow, which will be explained and used in Section 2 for a more general class of energies. The monotonicity of the $p$-Laplace operator allows to solve the approximating equations by Minty's trick alone. Nonetheless, to pass to the limit in the penalized equations some stronger compactness results on the $p$-Laplacien are needed. In the original work, this is based on the uniform monotonicity of the $p$-Laplace operator:

Theorem 2 For $k=1,2, \ldots$, let $f_{k}: M \times[0, T] \rightarrow \mathbb{R}^{l}$ satisfy the equation

$$
\partial_{t} f_{k}-\Delta_{p} f_{k}=g_{k}, \quad \text { on } M \times[0, T]
$$

in the sense of distributions. Assume that

1. $\left\{f_{k}\right\}_{k \in \mathbb{N}}$ is bounded in $L^{\infty}\left(0, T ; W^{1, p}\left(M, \mathbb{R}^{l}\right)\right)$,

2. $\left\{\partial_{t} f_{k}\right\}_{k \in \mathbb{N}}$ is bounded in $L^{2}\left(0, T ; L^{2}\left(M, \mathbb{R}^{l}\right)\right)$, and that

3. $\left\{g_{k}\right\}_{k \in \mathbb{N}}$ is bounded in $L^{1}\left(0, T ; L^{1}\left(M, \mathbb{R}^{l}\right)\right)$.

Then, $\left\{f_{k}\right\}_{k \in \mathbb{N}}$ is precompact in $L^{q}\left(0, T ; W^{1, q}\left(M, \mathbb{R}^{l}\right)\right)$ for each $1 \leq q<p$.

In Section 2 we offer a new approach to this compactness problem which involves some Young measure techniques, and which does not use strict or even uniform monotonicity properties of the operator. This allows in particular to obtain results for the flow of energies which are merely convex.

Theorem 1 was later proved for $1<p<2$ by Liu in [32], [33] and by Misawa in [37]. The $p$-harmonic flow from a unit ball in $\mathbb{R}^{m}$ into $S^{1} \subset \mathbb{R}^{2}$ was also considered by Courilleau and Demengel in [7]. There, also non-uniqueness of the $p$-harmonic flow with weakly $p$ harmonic, but not stationary harmonic initial data was proven, a result, however, which is already contained in [44] and even before that in [25].

The $p$-harmonic flow equation has two nonlinear terms: The $p$-Laplace term, and the right hand side involving the second fundamental form. The previously described compactness techniques allow to pass to the limit in the $p$-Laplace term only. The big problem, which is unsolved to the present day, is to pass to the limit in the nonlinear term on the right hand side of the equation. The sphere as target manifold has enough symmetries to rewrite the equations in such a way, that the right hand side simply vanishes (compare Section 2). A similar trick can be played to prove existence of the $p$-harmonic flow if the target manifold $N$ is a homogeneous space with a left invariant metric.

Theorem 3 ([23]) For $2<p$ there exists a global weak solution of the p-harmonic flow between Riemannian manifolds $M$ and $N$ for arbitrary initial data having finite p-energy in the case when the target $N$ is a homogeneous space with a left invariant metric. The solution $u: M \times[0, \infty[\rightarrow N$ satisfies the energy inequality

$$
\frac{1}{2} \int_{0}^{T} \int_{M}\left|\partial_{t} u\right|^{2} d t d \mu+\frac{1}{p} \int_{M}|D u(T)|^{p} d \mu \leq \frac{1}{p} \int_{M}|D u(0)|^{p} d \mu
$$

for almost all $T>0$. 
In this case of a homogeneous space as target, the equation of the $p$-harmonic flow can be reformulated such that the right hand side has a divergence structure (see [24] and [23]). In [23], a technique different from the penalization technique was used to approximate the equation, namely a time discretization. The reason is, that the penalization does not preserve the special structure of $N$.

The analogous result for $1<p<2$ was proven in [31]. There, the case of a modified $p$ energy is treated, namely that of the $p$-energy 'with potential', i.e. $E(u)=E_{p}-\int_{M} H(u) d \mu$ for a Lipshitz function $H$.

Another particular case which has been solved is the conformal case $p=m=\operatorname{dim} M$ :

Theorem 4 ([26]) For given initial value $u_{0} \in W^{1, m}(M, N)$ there exists a weak solution $u: M \times[0, \infty[\rightarrow N$ of the $m$-harmonic flow

$$
\begin{aligned}
u_{t}-\Delta_{m} u & \perp T_{u} N \\
u(\cdot, 0) & =u_{0} .
\end{aligned}
$$

$u$ satisfies the energy inequality (12) and is in $W^{1, m}(M)$ weakly continuous in time. There exists a set $\Sigma=\cup_{k=1}^{K} \Sigma_{k} \times\left\{T_{k}\right\}, \Sigma_{k} \subset M, 0<T_{k} \leq \infty$, such that on every open set $\Omega \subset M \times\left[0, \infty\left[\right.\right.$ with $\operatorname{dist}(\Omega,(M \times\{0\}) \cup \Sigma)=\mu>0$ there holds $\|\nabla u\|_{C^{0, \beta}(\Omega)} \leq C$ for some constants $C$ (depending on $m, E_{m}\left(u_{0}\right), M, N$ and $\mu$ ) and $\left.\beta \in\right] 0,1[$ (depending on $m, M$ and $N)$. There exists $\varepsilon_{1}>0$ such that the number $K$ of singular times is a priori bounded in terms of the initial energy, $K \leq \varepsilon_{1}^{-1} E_{m}\left(u_{0}\right)$, and the singular points $\left(x, T_{k}\right)$ are characterized by the condition $\lim \sup _{t} T_{k} E_{m}\left(u(t), B_{R}(x)\right) \geq \varepsilon_{1}$ for any $R>0$. At every singular time $T_{k}$ the decrease of the m-energy is at least $\varepsilon_{1}$ :

$$
E_{m}\left(u\left(T_{k}\right)\right) \leq \liminf _{t \nearrow T_{k}} E_{m}(u(t))-\varepsilon_{1} .
$$

In order to get rid of the nonlinear side condition, that the solution has to take values in the sphere, Hamilton's geometric technique from [20] was used, namely that of a total geodesic embedding of the target $N$ in some $\mathbb{R}^{k}$. I.e. the euclidean metric in $\mathbb{R}^{k}$ is deformed to a new metric $h$ such that

1. $N$ is still embedded isometrically,

2. the metric $h$ equals the Euclidean metric outside a large ball,

3. there exists an involutive isometry $\iota: T \rightarrow T$ on a tubular neighborhood $T$ of $N$ corresponding to multiplication by -1 in the orthonormal fibers of $N$ and having precisely $N$ for its fixed point set.

A $h$-geodesic curve $\gamma$ connecting $x, y \in N(x, y$ close enough) will always be contained in $N$. This follows from the (local) uniqueness of geodesics and the fact that with $\gamma$ the curve $\iota \circ \gamma$ is another geodesic joining $x$ and $y$.

The idea is that, if the initial data are smooth, then a short time solution $u$ of the $p$ harmonic flow is unique which forces it to stay in $N$ (since otherwise, $u$ and $\iota \circ u$ were two different solutions).

The crucial point in the conformal case is an energy concentration lemma: 
Theorem 5 ([26]) If $\operatorname{dim}(M)=p$ and $u \in C^{2}(M \times[0, T[; N)$ is a solution of the $m$ harmonic flow then there exist constants $c, \varepsilon_{0}>0$ which only depend on the geometry of the manifolds $M$ and $N$, and there exists a time $\left.T_{0} \in\right] 0, T[$ which depends in addition on $u_{0}$, with the following properties: If the initial local energy satisfies

$$
\sup _{x \in M} E_{m}\left(u(0), B_{2 R}(x)\right)<\varepsilon_{0}
$$

then it follows

$$
E_{m}\left(u(t), B_{R}(x)\right) \leq E_{m}\left(u(0), B_{2 R}(x)\right)+c E_{0}^{1-\frac{1}{p}} \frac{t}{R^{m}}
$$

for all $(x, t) \in M \times\left[0, T_{0}\right]$. Here $E_{0}$ denotes the initial energy, and $E_{m}(u(t), B)$ is the $m$-energy of $u(t)$ in a region $B \subset M$.

This lemma allows to control energy concentration of the flow for short time which leads to short time existence and (local) smoothness. At times, where the energy concentrates, the flow develops a singularity and can be restarted. It is not known whether for $m \neq 2$ the $m$-harmonic flow develops singularities in finite time, in contrast to the harmonic flow, where this is confirmed by the example of Chang, Ding and Ye in[4].

Technically, the proof of Theorem 4 is carried out by two approximation steps: In a first step, only smooth initial data are considered. At the same time, the $p$-energy is regularized by $E_{p, \varepsilon}=\int_{M}\left(\varepsilon+|D u|^{2}\right)^{p / 2} d \mu$. In a second step, arbitrary initial data are approximated by smooth functions (observe, that by Bethuel-Zheng [3] $C^{\infty}(M, N)$ is dense in $W^{1, p}(M, N)$ ). Misawa improved Theorem 4 in [34] by showing, that, as it is the case for the harmonic flow, the energy concentration set $\Sigma$ is discrete not only in time, but also in space.

The $p$-harmonic flow in the case of non-positive sectional curvature of the target manifold was treated by Fardoun and Regbaoui in [14] and [16]:

Theorem 6 If $\operatorname{Riem}_{N} \leq 0$ and $u_{0} \in C^{2, \alpha}(M, N)$, then for $p>1$ :

1. There exists a unique global weak solution $u$ of the p-harmonic flow from $M$ to $N$ such that $\partial_{t} u \in L^{2}\left(\left[0, \infty[\times M)\right.\right.$ and $u, D u \in C^{\beta}([0, \infty[\times M)$ for some $\beta \in] 0,1[$. Moreover, the solution satisfies the energy inequality (12) for all $t>0$.

2. There exists a sequence $t_{k} \rightarrow \infty$ such that $u\left(t_{k}, \cdot\right)$ converges in $C^{1, \beta^{\prime}}(M, N)$, for all $\beta^{\prime}<\beta$, to a weakly p-harmonic map $u_{\infty} \in C^{1, \beta}(M, N)$.

In particular, this results settles the homotopy question in this case. The line of proof goes along regularization of the $p$-energy, and a Bochner formula for the energy density (see [14] for details).

The same result for $p>2$ was shown earlier by Misawa in [35] under the assumption that the image of $u_{0}$ is contained in a geodesic ball, and in [36] without that latter assumption.

The $p$-harmonic flow for the case of small initial data has been treated by Fardoun and Regbaoui in [15]:

Theorem 7 For any $p>1$ and $K>0$ there exists an $\varepsilon_{0}>0$ depending on $K, M, N$ and $p$ such that if $u_{0} \in C^{2, \alpha}(M, N), 0<\alpha<1$, with $E_{p}\left(u_{0}\right) \leq \varepsilon_{0}$ and $\left\|D u_{0}\right\|_{L^{\infty}}(M) \leq K$, the following is true: 
1. There exists a unique global weak solution $u$ of the p-harmonic flow from $M$ to $N$ such that $\partial_{t} u \in L^{2}\left(\left[0, \infty[\times M)\right.\right.$ and $u, D u \in C^{\beta}([0, \infty[\times M)$ for some $\beta \in] 0,1[$. Moreover, the solution satisfies the energy inequality (12) for all $t>0$.

2. There exists a sequence $t_{k} \rightarrow \infty$ such that $u\left(t_{k}, \cdot\right)$ converges in $C^{1, \beta^{\prime}}(M, N)$, for all $\beta^{\prime}<\beta$, to a weakly p-harmonic map $u_{\infty} \in C^{1, \beta}(M, N)$. Moreover there exists $\bar{\varepsilon}_{0}>0$ depending on $K, M, N$ and $p$ such that if in addition $E_{p}\left(u_{0}\right) \leq \bar{\varepsilon}_{0}$, then $u_{\infty}$ is a constant map.

This result of Fardoun and Regbaoui concludes our survey on the $p$-harmonic flow.

\section{Heat flow into spheres for a class of energies}

In this section, our target manifold $N$ is the unit sphere $S^{n} \subset \mathbb{R}^{n+1}$. In order to keep the formulas short, we restrict this presentation to the case $M=T=\mathbb{R}^{m} / \mathbb{Z}^{m}$ of a flat torus as domain manifold. The modifications necessary for a general compact smooth Riemannian manifold without boundary are purely technical and straightforward.

The class of energy functionals we want to investigate here is of the form

$$
E(u)=\int_{T} \rho(|D u|) d x
$$

for functions $u: T \rightarrow S^{n} \subset \mathbb{R}^{n+1}$. Here $\rho: \mathbb{R}_{\geq 0} \rightarrow \mathbb{R}$ is supposed to be continuously differentiable, convex, and satisfies the coercivity and growth conditions

$$
\begin{aligned}
C_{1} \xi^{p} & \leq \rho(\xi) & & \text { for all } \xi \geq 0 \\
0 & \leq \rho^{\prime}(\xi) \leq C_{2} \xi^{p-1} & & \text { for all } \xi \geq 0
\end{aligned}
$$

for two positive constants $C_{1}$ and $C_{2}$, and for a given $p \geq 2$. The energy flow related to this energy is described by the following equation

$$
\partial_{t} u-\operatorname{div}\left(\rho^{\prime}(|D u|) \frac{D u}{|D u|}\right) \quad \perp \quad T_{u} S .
$$

We use the shorthand notation

$$
\sigma(D u):=\rho^{\prime}(|D u|) \frac{D u}{|D u|}
$$

and hence the previous equation can be rewritten in the form

$$
\partial_{t} u-\operatorname{div} \sigma(D u)=\lambda u
$$

for a function $\lambda(x, t)$. Observe, that by multiplying this equation by $u$, we find

$$
\lambda=\sigma(D u): D u \text {. }
$$

Here, $A: B:=\operatorname{trace}\left(A B^{\mathrm{T}}\right)$ denotes the usual inner product of two matrices of the same type. So, the final form of the heat equation we want to solve, is

$$
\begin{aligned}
\partial_{t} u-\operatorname{div} \sigma(D u) & =u \sigma(D u): D u & & \text { on } T \times \mathbb{R}_{>0} \\
u(\cdot, 0) & =u_{0} & & \text { on } T .
\end{aligned}
$$

We suppose, that the initial data $u_{0}$ have finite energy, i.e. $u_{0} \in W^{1, p}\left(T, S^{n}\right)$. 


\subsection{Approximation by penalization}

We construct a family of approximating problems by the classical penalization method. To this end, we consider the energies

$$
E_{k}(u):=E(u)+k \int_{T} \chi(|u|) d x
$$

for functions $u: T \rightarrow \mathbb{R}^{n+1}$, and for $k \in \mathbb{N}$. The smooth function $\chi: \mathbb{R}_{\geq 0} \rightarrow \mathbb{R}$ is chosen in such a way, that $0 \leq \chi \leq 1, \chi=1$ outside the interval $\left[\frac{1}{2}, \frac{3}{2}\right], \chi(x)=0$ if and only if $x=1$, and that $\chi^{\prime}$ changes sign only once (see Figure 1 ). The idea is, that we abandon

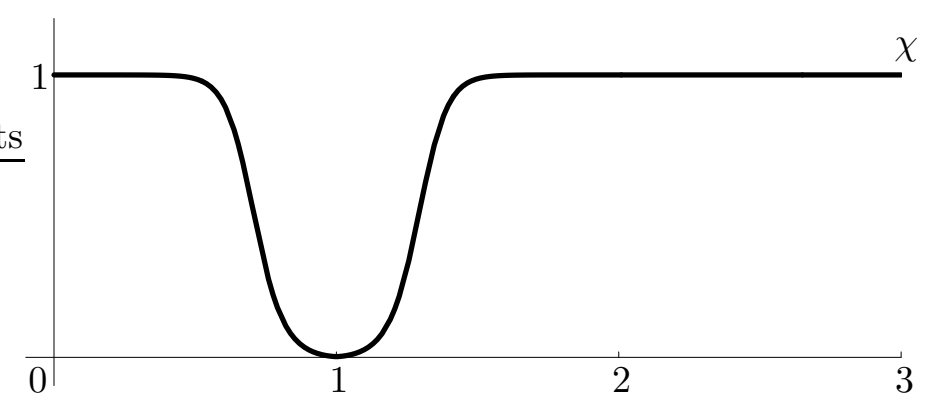

Figure 1: The function $\chi$

the restriction that $u$ takes values in the target $S^{n}$, but that (in terms of energy) with increasing $k$, it becomes more and more favorable for the function $u$ to take values close to the sphere $S^{n}$.

The heat flow equation for the penalized energy is

$$
\partial_{t} u-\operatorname{div} \sigma(D u)+k \chi^{\prime}(|u|) \frac{u}{|u|}=0 .
$$

The goal is now to prove existence of a global weak solution for this penalized energy flow with the given initial data (17).

Lemma 8 Let $k \in \mathbb{N}$ and $u_{0} \in W^{1, p}\left(T, S^{n}\right)$. Then, (17)-(18) possesses a weak solution $u_{k} \in L^{\infty}\left(0, \infty ; W^{1, p}\left(T, \mathbb{R}^{n+1}\right)\right)$ which is subject to the energy inequality

$$
\int_{0}^{\tau}\left\|\partial_{t} u_{k}\right\|_{L^{2}(T)}^{2} d t+E_{k}\left(u_{k}(\tau)\right) \leq E\left(u_{0}\right)
$$

for almost all $\tau \geq 0$.

Proof: $W^{1, p}\left(T, \mathbb{R}^{n+1}\right)$ is separable and possesses therefore a Galerkin base $\left\{w_{i}\right\}_{i \in \mathbb{N}}$, $w_{i} \in W^{1, p}\left(T, \mathbb{R}^{n+1}\right)$ in the sense, that for every $w \in W^{1, p}\left(T, \mathbb{R}^{n+1}\right)$ there exist coefficients $c_{i}^{(j)}$ such that

$$
w^{(j)}:=\sum_{i=1}^{j} c_{i}^{(j)} w_{i} \rightarrow w \quad \text { in } W^{1, p}(T) \text { as } j \rightarrow \infty .
$$


It is convenient to choose the $w_{i}$ smooth and $L^{2}$-orthonormal. For fixed $j \in \mathbb{N}$, we make the ansatz

$$
u^{(j)}(t):=\sum_{i=1}^{j} c_{i}^{(j)}(t) w_{i}
$$

for a solution of the approximating system

$$
\int_{T}\left(\partial_{t} u^{(j)} w_{l}+\sigma\left(D u^{(j)}\right): D w_{l}+k \chi^{\prime}\left(\left|u^{(j)}\right|\right) \frac{u^{(j)}}{\left|u^{(j)}\right|} w_{l}\right) d x=0 \quad \text { for } l=1, \ldots, j
$$

with initial values

$$
u^{(j)}(0)=u_{0}^{(j)} \rightarrow u_{0} \quad \text { in } W^{1, p}(T) \text { for } j \rightarrow \infty .
$$

This is a system of $j$ ordinary differential equations for the coefficients $c_{l}^{(j)}(t), l=1, \ldots, j$. It is easy to verify that this system satisfies the hypotheses of the existence theorem of Picard-Lindelöf and that therefore a solution exists on a time interval $] 0, \tau(j)[$. Since, by construction, $u^{(j)}$ is an element of $\operatorname{span}\left(w_{1}, \ldots, w_{j}\right)$, equation (20) holds with $u^{(j)}$ in place of $w_{l}$, and hence, after integration over a time interval $] 0, \tau[, \tau \leq \tau(j)$, one gets

$$
\begin{aligned}
\frac{1}{2}\left\|u^{(j)}(\tau)\right\|_{L^{2}(T)}^{2}+\int_{0}^{\tau} \int_{T} \sigma\left(D u^{(j)}\right) & : D u^{(j)} d x d t+ \\
& +\int_{0}^{\tau} \int_{T} k \chi^{\prime}\left(\left|u^{(j)}\right|\right) \frac{u^{(j)}}{\left|u^{(j)}\right|} u^{(j)} d x d t=\frac{1}{2}\left\|u^{(j)}(0)\right\|_{L^{2}(T)}^{2}
\end{aligned}
$$

The first term in (22) is equal to $\frac{1}{2}\left|c^{(j)}(\tau)\right|_{\mathbb{R}^{j}}^{2}$. By (15), the second term is non-negative. Since $\left|\chi^{\prime}(t)\right| t \leq \frac{3}{2} \max \left|\chi^{\prime}\right|$ for $t \geq 0$, the third term, in absolute value, is bounded by $C \tau$ for a constant $C$. This shows, that the functions $c_{j}^{(j)}(t)$ are bounded on $[0, \tau(j)[$, and hence, by (20), the same is true for $\frac{d}{d t} c_{j}^{(j)}(t)$. Therefore, the existence interval is both open and closed and hence we obtain a global solution $u^{(j)}$ of $(20)$.

Similarly, with $\partial_{t} u^{(j)}$ in place of $w_{l}$ in (20), we obtain

$$
\int_{0}^{\tau}\left\|\partial_{t} u^{(j)}\right\|_{L^{2}(T)}^{2} d t+E_{k}\left(u^{(j)}(\tau)\right)=E_{k}\left(u_{0}^{(j)}\right) \leq C \quad \text { for all } j \text { and } \tau \geq 0 .
$$

Observe, that

$$
E_{k}(u) \geq E(u)=\int_{T} \rho(|D u|) d x \geq C_{1} \int_{T}|D u|^{p} d x
$$

by the coercivity assumption (14) on $\rho$. Therefore, by (23), the sequence $\left\{u^{(j)}\right\}_{j}$ is bounded in $L^{\infty}\left(0, \infty ; W^{1, p}(T)\right)$ On the other hand, still by $(23)$, the sequence $\left\{\partial_{t} u^{(j)}\right\}_{j}$ is bounded in $L^{2}(T \times] 0, \infty[)$. Aubin's lemma therefore implies that there exists a subsequence $\left\{u^{\left(j^{\prime}\right)}\right\}_{j^{\prime}}$ and a function $u$ such that

$$
\left.u^{\left(j^{\prime}\right)} \rightarrow u \quad \text { in } L^{r}(T \times] 0, \tau[) \text { for all } \tau \in\right] 0, \infty\left[\text { and all } r \in \left[p, \frac{m p}{m-p}[.\right.\right.
$$

Notice that to have one subsequence which works for all such $\tau$ and $r$, one can apply the usual diagonal sequence technique. By passing to a further subsequence if necessary, we can also assume that

$$
\left.u^{\left(j^{\prime}\right)} \rightarrow u \quad \text { point-wise almost everywhere on } T \times\right] 0, \infty[
$$


and

$$
u^{\left(j^{\prime}\right)} \rightarrow u \quad \text { weakly* in } L^{\infty}\left(0, \infty ; W^{1, p}\left(T, \mathbb{R}^{n+1}\right)\right)
$$

and finally, that

$$
\partial_{t} u^{(j)} \rightarrow \partial_{t} u \quad \text { weakly in } L^{2}(T \times] 0, \infty[) .
$$

Notice also, that by the growth condition (15) we imposed on $\rho$, the sequence $\left\{\sigma\left(D u^{(j)}\right)\right\}_{j}$ is bounded in $L^{\infty}\left(0, \infty ; L^{p^{\prime}}(T)\right)$. Therefore,

$$
\sigma\left(D u^{\left(j^{\prime}\right)}\right) \rightarrow w \quad \text { weakly* in } L^{\infty}\left(0, \infty ; L^{p^{\prime}}(T)\right)
$$

for a further subsequence.

For a test function $\phi(x, t)=\xi(t) \eta(x)$, with $\xi \in C_{0}^{\infty}(] 0, \infty[)$ and $\eta \in \operatorname{span}\left(w_{1}, \ldots, w_{l}\right)$, we infer from (20) that (as soon as $j^{\prime}>l$ )

$$
\begin{array}{cccc}
\int_{0}^{\infty} \int_{T} \partial_{t} u^{\left(j^{\prime}\right)} \phi & +\int_{0}^{\infty} \int_{T} \sigma\left(D u^{\left(j^{\prime}\right)}\right): D \phi & +\int_{0}^{\infty} \int_{T} k \chi^{\prime}\left(\left|u^{\left(j^{\prime}\right)}\right|\right) \frac{u^{\left(j^{\prime}\right)}}{\left|u^{\left(j^{\prime}\right)}\right|} \phi & =0 \\
\downarrow & \downarrow & \downarrow \text { as } j^{\prime} \rightarrow \infty & \\
\int_{0}^{\infty} \int_{T} \partial_{t} u \phi & +\int_{0}^{\infty} \int_{T} w: D \phi & +\int_{0}^{\infty} \int_{T} k \chi^{\prime}(|u|) \frac{u}{|u|} \phi & =0
\end{array}
$$

To pass to the limit in the first and the second term of (29), we used (27) and (28). For the third term, we used (25) and Lebesgue's dominated convergence theorem. By density of the linear span of testfunctions we used, the resulting equation in (29) follows also for arbitrary $\phi \in C_{0}^{\infty}(T \times] 0, \infty[)$.

In order to identify $w$ we now want to apply Minty's lemma for monotone operators. (see, e.g. [42, "decisive monotonicity trick"]). To do this, we first observe, that by (22), for $j^{\prime} \rightarrow \infty$, and by using (24), (25) and (27)

$$
\begin{aligned}
\int_{0}^{\tau} \int_{T}-\sigma\left(D u^{\left(j^{\prime}\right)}\right): D u^{\left(j^{\prime}\right)}=\int_{0}^{\tau} \int_{T} \partial_{t} u^{\left(j^{\prime}\right)} u^{\left(j^{\prime}\right)} & +\int_{0}^{\tau} \int_{T} k \chi^{\prime}\left(\left|u^{\left(j^{\prime}\right)}\right|\right) \frac{u^{\left(j^{\prime}\right)}}{\left|u^{\left(j^{\prime}\right)}\right|} u^{\left(j^{\prime}\right)} \rightarrow \\
& \rightarrow \int_{0}^{\tau} \int_{T} \partial_{t} u u+\int_{0}^{\tau} \int_{T} k \chi^{\prime}(|u|) \frac{u}{|u|} u .
\end{aligned}
$$

On the other hand, by (29), we have

$$
\int_{0}^{\tau} \int_{T}-w: D u=\int_{0}^{\tau} \int_{T} u \partial_{t} u+\int_{0}^{\tau} \int_{T} k \chi^{\prime}(|u|) \frac{u}{|u|} u
$$

We now apply Minty's lemma for the monotone operator

$$
\begin{aligned}
A: L^{p}\left(0, \tau ; W^{1, p}(T)\right) & \rightarrow L^{p^{\prime}}\left(0, \tau ; W^{-1, p^{\prime}}(T)\right) \\
u & \mapsto\left(\phi \mapsto \int_{0}^{\tau} \int_{T} \sigma(D u): D \phi\right) .
\end{aligned}
$$

Now, as desired, from (26), (28), and the fact that the right hand sides of (30) and (31) agree, it follows from Minty's lemma that $w=\sigma(D u)$. Hence, $u$ is a weak solution of (18).

The energy inequality (19) for $u$ follows from (23) as $j \rightarrow \infty$ : We have

$$
\liminf _{j \rightarrow \infty} \int_{0}^{\tau}\left\|\partial_{t} u^{(j)}\right\|_{L^{2}(T)}^{2} \geq \int_{0}^{\tau}\left\|\partial_{t} u\right\|_{L^{2}(T)}^{2}
$$


by $(27)$,

$$
\liminf _{j \rightarrow \infty} E_{k}\left(u^{(j)}(\tau)\right) \geq E_{k}(u(\tau)) \quad \text { for almost all } \tau
$$

by (26), the convexity of $\rho$, and by (25). And finally, observe that

$$
\lim _{j \rightarrow \infty} E_{k}\left(u_{0}^{(j)}\right)=E_{k}\left(u_{0}\right)
$$

by (21), the Vitali convergence theorem and by (25).

\subsection{Passage to the limit I}

We now want to pass to the limit in (18). Observe first, that by the energy inequality (19), the sequence $\left\{u_{k}\right\}$ we constructed is bounded in $L^{\infty}\left(0, \infty ; W^{1, p}(T)\right)$ and $\left\{\partial_{t} u_{k}\right\}$ is bounded in $L^{2}\left(0, \infty ; L^{2}(T)\right)$. By passing to a subsequence which we still denote by $u_{k}$, we may assume that

$$
u_{k} \rightarrow u \quad \text { weakly* in } L^{\infty}\left(0, \infty ; W^{1, p}(T)\right)
$$

and

$$
\partial_{t} u_{k} \rightarrow \partial_{t} u \quad \text { weakly in } L^{2}(T \times] 0, \infty[) .
$$

Moreover, by Aubin's Lemma, we can extract a further subsequence such that

$$
\left.u_{k} \rightarrow u \text { in } L^{r}(T \times] 0, \tau[) \text { for all } \tau \in\right] 0, \infty\left[\text { and all } r \in \left[p, \frac{m p}{m-p}[,\right.\right.
$$

and hence, for yet a further subsequence

$$
\left.u_{k} \rightarrow u \quad \text { point-wise almost everywhere on } T \times\right] 0, \infty[
$$

Notice, that from the energy inequality (19) it follows, that $\left|u_{k}\right| \rightarrow 1$ almost everywhere. Combining this with (35), we conclude, that $|u|=1$ almost everywhere on $T \times[0, \infty[$, i.e. $u$ takes values in the sphere, as desired. This is, nonetheless, not sufficient to already pass to the limit in (18).

The following lemma is a maximum principle for the weak solutions $u_{k}$ of (18).

Lemma 9 Let $u_{k}$ be a weak solution of (18) on $\left[0, \tau\left[\right.\right.$. Then, $\left|u_{k}(t)\right| \leq 1$ for all $t \in[0, \tau[$, if $\left|u_{k}(0)\right| \leq 1$.

Proof: To ease notation, we write $u$ in place of $u_{k}$. We use

$$
R(u):=u-\frac{u}{|u|} \min \{1,|u|\}
$$

as a test function in (18) and find for the three single terms:

$$
\int_{T} R(u) \partial_{t} u=\partial_{t} \int_{T} \xi(|u|)
$$

for

$$
\xi(x)= \begin{cases}0 & \text { if } 0 \leq x<1 \\ \frac{1}{2}(x-1)^{2} & \text { if } x \geq 1\end{cases}
$$


Therefore

$$
\int_{T} R(u) \partial_{t} u=\partial_{t} \int_{\{|u| \geq 1\}}(|u|-1)^{2}
$$

In the second term, we get

$$
\int_{T} k \chi^{\prime}(|u|) \frac{u}{|u|} R(u) \geq 0
$$

since $\chi^{\prime}(|u|) \geq 0$ for $|u| \geq 1$ and $R(u)=0$ for $|u| \leq 1$. For the third term, we have

$$
\int_{T} \sigma(D u): D R(u)=\int_{\{|u| \geq 1\}} \sigma(D u): D u\left(1-\frac{1}{|u|}\right)+\int_{\{|u| \geq 1\}} \rho^{\prime}(|D u|) \frac{|u D u|^{2}}{|D u||u|^{3}}
$$

Observe, that the last term in the previous expression is non-negative. Hence, summarizing, we obtain

$$
\frac{1}{2} \frac{\partial}{\partial t} \int_{\{|u| \geq 1\}}(|u|-1)^{2}+\int_{\{|u| \geq 1\}} \sigma(D u): D u\left(1-\frac{1}{|u|}\right) \leq 0 .
$$

Since the second term is non-negative, the derivative in the first term is non-positive, and therefore the lemma follows.

By (31), we have for arbitrary $\tau>0$

$$
\int_{0}^{\tau} \int_{T} \partial_{t} u_{k} u_{k}+\int_{0}^{\tau} \int_{T} \sigma\left(D u_{k}\right): D u_{k}+k \int_{0}^{\tau} \int_{T} \chi^{\prime}\left(\left|u_{k}\right|\right) \frac{u_{k}}{\left|u_{k}\right|} u_{k}=0 .
$$

The energy inequality (23) and the growth condition (15) imply, that the first two terms are, for fixed $\tau$, uniformly bounded in $k$. In view of Lemma 9, and the fact, that $\chi^{\prime}$ vanishes on $\left[0, \frac{1}{2}\right]$ we obtain

$$
\int_{0}^{\tau} \int_{T}\left|k \chi^{\prime}\left(\left|u_{k}\right|\right) \frac{u_{k}}{\left|u_{k}\right|}\right| \leq-2 \int_{0}^{\infty} \int_{T} k \chi^{\prime}\left(\left|u_{k}\right|\right) \frac{u_{k}}{\left|u_{k}\right|} u_{k} \leq C
$$

for a constants $C$ which does not depend on $k$.

We conclude, that the right hand side in

$$
\partial_{t} u_{k}-\operatorname{div} \sigma\left(D u_{k}\right)=-k \chi^{\prime}\left(\left|u_{k}\right|\right) \frac{u_{k}}{\left|u_{k}\right|}
$$

is a bounded sequence in $L^{1}(T \times[0, \tau])$. This fact suggests now to consider the Young measure generated by the sequence $u_{k} \otimes D u_{k}$. Observe, that $u_{k}$ converges almost everywhere, an therefore, if $D u_{k}$ generates the Young measure $\nu$ on the space $\mathbb{I}^{(n+1) \times m}$ of $(n+1) \times m$ matrices, then $u_{k} \otimes D u_{k}$ generates the Young measure $\delta_{u} \otimes \nu$ (see, e.g., [27]). We use the next section to collect some relevant facts about the Young measure $\nu$.

\subsection{The Young measure generated by $D u_{k}$}

Lemma 10 Let $\nu$ be the Young measure generated by the the gradients Du $u_{k}$ of the solutions of (18) constructed in Lemma 8. Then

(i) $\nu_{(x, t)}$ is a probability Young measure for almost every $(x, t) \in T \times[0, \tau]$. 
(ii) $\nu_{(x, t)}$ satisfies $D u(x, t)=\left\langle\nu_{(x, t)}\right.$, id $\rangle$ for almost every $(x, t) \in T \times[0, \tau]$.

Proof: (i) follows directly from the fact, that $D u_{k}$ is bounded in $L^{1}(T \times[0, \tau])$ (see, e.g., [1] or $[27])$.

(ii): $D u_{k}$ converges weakly to $D u$ in $L^{p}(T \times[0, \tau])$. On the other hand, since $D u_{k}$ is an equiintegrable sequence, we have that $D u_{k}$ converges weakly to $\left\langle\nu_{(x, t)}\right.$, id $\rangle$ in $L^{1}(T \times[0, \tau])$. Therefore, $D u(x, t)=\left\langle\nu_{(x, t)}, \mathrm{id}\right\rangle$.

The final ingredient we need is a "div-curl inequality":

$$
\int_{0}^{\tau} \int_{T} \int_{\mathbb{M}^{(n+1) \times m}}(\sigma(\lambda)-\sigma(D u)):(\lambda-D u) d \nu_{(x, t)}(\lambda) d x d t \leq 0
$$

This inequality appears first in [8] for the Young measure $\nu$ generated by sequence of the gradients of solutions of a stationary non-linear elliptic system of $p$-Laplace type with right hand side bounded in $L^{1}$. The class of operators considered there includes in particular our case $\sigma(D u)$. Due to the fact that, as we have seen, the right hand side in (36) is bounded in $L^{1}$, the proof in [8] carries over to the parabolic case.

\subsection{Passage to the limit II}

The aim is now, to pass to the limit in the penalized equation (18). The problem in the operator term is, that $\rho$ need not be strictly convex, and hence $\sigma$ is not necessarily strictly monotone, or even uniformly monotone like in the case of the $p$-Laplace operator. Nonetheless, if $\rho$ is not strictly convex in a region, it must be affine there, and it should therefore nevertheless be possible to pass to the limit there. It turns out that Young measures provide the adequate tool to go through with this program.

We start with some notation.

We consider the limit $u$ of $u_{k}$ as in $(32)-(35)$. Let $(x, t)$ be a fixed point in $T \times[0, \tau]$. Then, let

$L:=\left\{(\lambda, \rho(|D u(x, t)|)+\sigma(D u(x, t)):(\lambda-D u(x, t))) \mid \lambda \in \mathbb{M}^{(n+1) \times m}\right\} \subset M^{(n+1) \times m} \times \mathbb{R}$

denote the supporting hyperplane to the graph of $\rho(|\lambda|)$ in $(D u(x, t), \rho(|D u(x, t)|))$ (see Figure 2). Moreover, let

$$
K_{(x, t)}=\left\{\lambda \in \mathbb{M}^{(n+1) \times m} \mid \rho(|\lambda|)=\rho(|D u(x, t)|)+\sigma(D u(x, t)):(\lambda-D u(x, t))\right\}
$$

denote the set, where $\rho$ agrees with the supporting hyperplane $L$ (see Figure 2).

Lemma 11 For almost all $(x, t) \in T \times[0, \tau]$, the Young measure $\nu_{(x, t)}$ is supported in the set $K_{(x, t)}$, i.e. $\operatorname{spt}\left(\nu_{(x, t)}\right) \subset K_{(x, t)}$.

Proof: By (37) the integrand $(\sigma(\lambda)-\sigma(D u(x, t))):(\lambda-D u(x, t))$ (which is non-negative everywhere because of the monotonicity of $\sigma$ ) must vanish as a function of $\lambda$ on the support of the measure $\nu_{(x, t)}$. This is true with the possible exception of a set $N$ of $(x, t) \in T \times[0, \tau]$ of measure zero. Let us fix $\lambda \in \operatorname{spt}\left(\nu_{(x, t)}\right)$ for $(x, t) \notin N$, then

$$
(1-\alpha)(\sigma(D u(x, t))-\sigma(\lambda)):(D u(x, t)-\lambda)=0 \quad \text { for all } \alpha \in[0,1] .
$$




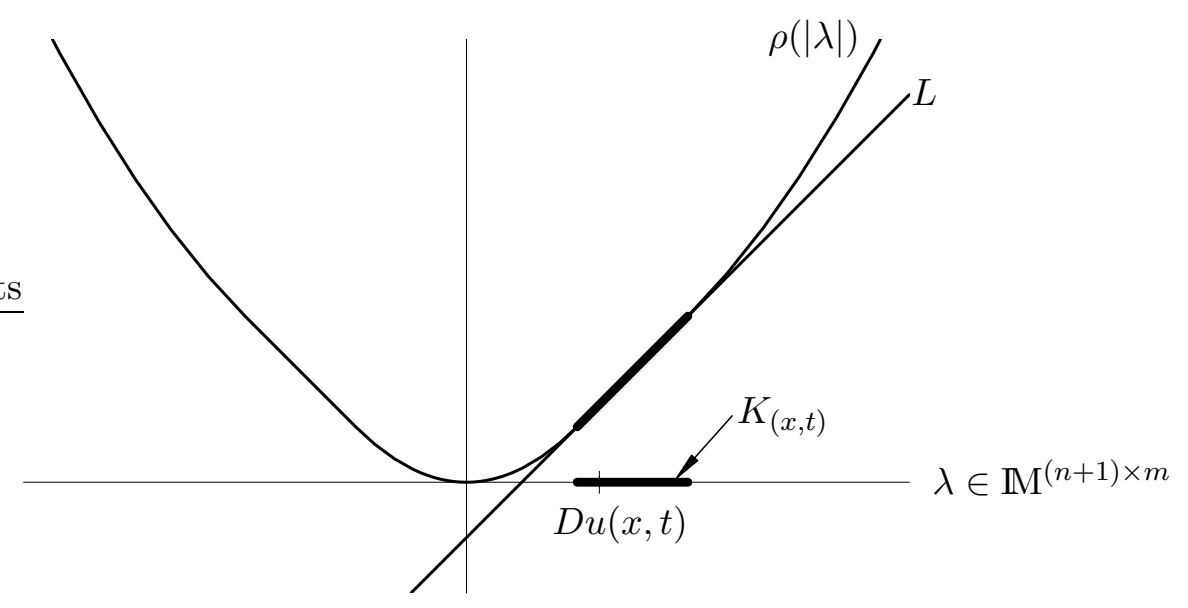

Figure 2: Graph of $\rho$

On the other hand, again by the monotonicity of $\sigma$, we have

$$
0 \leq(1-\alpha)(\sigma(D u(x, t)+\alpha(\lambda-D u(x, t)))-\sigma(\lambda)):(D u(x, t)-\lambda) \quad \text { for all } \alpha \in[0,1] .
$$

Subtracting (38) from (39) we obtain

$0 \leq(1-\alpha)(\sigma(D u(x, t)+\alpha(\lambda-D u(x, t)))-\sigma(D u(x, t))):(D u(x, t)-\lambda) \quad$ for all $\alpha \in[0,1]$.

But by monotonicity, also the opposite inequality holds true:

$0 \geq(1-\alpha)(\sigma(D u(x, t)+\alpha(\lambda-D u(x, t)))-\sigma(D u(x, t))):(D u(x, t)-\lambda) \quad$ for all $\alpha \in[0,1]$.

Therefore, still for the same $\lambda \in \operatorname{spt}\left(\nu_{(x, t)}\right)$, by (40) and (41), we have

$0=(1-\alpha)(\sigma(D u(x, t)+\alpha(\lambda-D u(x, t)))-\sigma(D u(x, t))):(D u(x, t)-\lambda) \quad$ for all $\alpha \in[0,1]$.

Using (42), we conclude

$$
\begin{aligned}
\rho(|\lambda|) & =\rho(|D u(x, t)|)+\int_{0}^{1} \sigma(D u(x, t)+\alpha(\lambda-D u(x, t)):(\lambda-D u(x, t)) d \alpha= \\
& =\rho(|D u(x, t)|)+\sigma(D u(x, t)):(\lambda-D u(x, t))
\end{aligned}
$$

and hence, $\lambda \in K_{(x, t)}$, as claimed.

Lemma 12 (i) For each $\lambda \in K_{(x, t)}$ there holds $\sigma(\lambda)=\sigma(D u(x, t))$.

(ii) For almost all $(x, t) \in T \times[0, \tau]$ the following is true:

$$
\int_{M^{(n+1) \times m}} \sigma(\lambda) d \nu_{(x, t)}(\lambda)=\sigma(D u(x, t)) .
$$

Proof: (i) follows the fact that $\rho$ is continuously differentiable.

(ii): As we have seen in Lemma 11, the support of the measure $\nu_{(x, t)}$ is contained in the set $K_{(x, t)}$. Therefore, in (43), we only need to integrate over $K_{(x, t)} \subset M^{(n+1) \times m}$. But 
there, as stated in (i), $\sigma(\lambda)=\sigma(D u(x, t))$. Hence, the claim follows from the fact that, by Lemma 10(i), $\nu_{(x, t)}$ has mass 1.

Actually, from (ii) in the previous lemma and the fundamental theorem on Young measures (see, e.g., [1]) it now already follows, that $\sigma\left(D u_{k}\right) \rightarrow \sigma(D u)$ in $L^{1}(T \times[0, \tau]$. However, as we will see in the next lemma, this convergence is even strong.

Lemma 13 Let $u_{k}$ be the sequence solutions of (18) constructed in Lemma 8, u the limit of this sequence in (32), and

$$
g_{k}(x, t):=\left|\sigma\left(D u_{k}(x, t)\right)-\sigma(D u(x, t))\right|
$$

Then, $g_{k} \rightarrow 0$ in $L^{1}(T \times[0, \tau])$ for all $\tau>0$.

Proof: Observe, that, since $\left\{D u_{k}\right\}$ is bounded in $L^{\infty}\left(0, \infty ; L^{p}(T)\right)$, by $(15) \sigma\left(D u_{k}\right)$ is bounded in $L^{\infty}\left(0, \tau ; L^{p^{\prime}}(T)\right)$. Therefore, $g_{k}$ is an equiintegrable sequence on $T \times[0, \tau]$. Hence, by the fundamental theorem of Young measures (see, e.g., [1]), for a suitable (not relabeled) subsequence, $g_{k} \rightarrow \bar{g}$ in $L^{1}(T \times[0, \tau])$ where

$$
\bar{g}(x, t)=\int_{\mathbb{M}^{(n+1) \times m}}|\sigma(\lambda)-\sigma(D u(x, t))| d \nu_{(x, t)}=0
$$

Here, we have used that by Lemma 11, we only have to integrate over $K_{(x, t)}$ and that there, by Lemma $12(\mathrm{i}), \sigma(\lambda)=\sigma(D u(x, t))$. Since $g_{k} \geq 0$, the convergence is strong. By the usual diagonal sequence argument, we can find a subsequence which converges strongly in $L_{\mathrm{loc}}^{1}(T \times[0, \infty])$.

This allows us now to finally prove the existence theorem for the heat flow of the energy $E$ :

Theorem 14 If the convex $C^{1}$-function $\rho$ satisfies the coercivity and growth conditions (14) and (15), then there exists a global weak solution of (16) for arbitrary initial data $u_{0} \in$ $W^{1, p}\left(T, S^{n}\right)$.

Proof: It follows from Lemma 13, that

$$
\sigma\left(D u_{k}\right) \rightarrow \sigma(D u) \quad \text { in } L_{\mathrm{loc}}^{1}(T \times[0, \infty])
$$

By taking the wedge product of (18) with $u_{k}$, we find that

$$
0=\partial_{t} u_{k} \wedge u_{k}-\operatorname{div}\left(\rho^{\prime}\left(\left|D u_{k}\right|\right) \frac{D u_{k}}{\left|D u_{k}\right|} \wedge u_{k}\right) .
$$

Passing to the limit in the weak form of (45), and using (33), (34) and (44) yields

$$
0=\partial_{t} u \wedge u-\operatorname{div}\left(\rho^{\prime}(|D u|) \frac{D u}{|D u|} \wedge u\right) .
$$

As stated at the beginning of Section 2.2, $|u|=1$ almost everywhere on $T \times[0, \infty[$. Therefore,

$$
\left(\partial_{t} u-\operatorname{div}(\sigma(D u))-u \sigma(D u): D u\right) u \psi=0
$$

is an identity for arbitrary $\psi \in C_{0}^{\infty}(T \times[0, \infty])$. 
Let $\phi \in C_{0}^{\infty}\left(T \times[0, \infty], \mathbb{R}^{n+1}\right)$. We test (46) by $u \wedge \phi$ and use $\psi=u \cdot \phi$ in (47) (observe, that these are admissible test-functions) and subtract the resulting equations from each another. Using the identity

$$
\phi=u(u \cdot \phi)-u \wedge(u \wedge \phi)
$$

this yields the weak form of (16).

\section{References}

[1] J. M. Ball: A version of the fundamental theorem for Young measures. In: Partial differential equations and continuum models of phase transitions: Proceedings of an NSF-CNRS joint seminar. Springer, 1989

[2] F. Bethuel: The approximation problem for Sobolev maps between manifolds. Acta Math. 167, 153-206 (1991)

[3] F. Bethuel, X. Zheng: Density of smooth functions between two manifolds in Sobolev spaces. J. Funct. Anal. 80, 60-75 (1988)

[4] K.-C. Chang, W.-Y. Ding, R. Ye, R.: Finite-time blow-up of the heat flow of harmonic maps from surfaces. J. Differ. Geom. 36, No. 2, 507-515 (1992)

[5] Y. Chen: The weak solutions to the evolution problem of harmonic maps. Math. Z. 201, 69-74 (1989)

[6] Y. Chen, M.-C. Hong, N. Hungerbühler: Heat flow of $p$-harmonic maps with values into spheres. Math. Z. 215, 25-35 (1994)

[7] P. Courilleau, F. Demengel: Heat flow for $p$-harmonic maps with values in the circle. Nonlinear Anal. 41, no. 5-6, Ser. A: Theory Methods, 689-700 (2000)

[8] G. Dolzmann, N. Hungerbühler, S. Müller: Non-linear elliptic systems with measurevalued right hand side. Math. Z. 226, 545-574 (1997)

[9] F. Duzaar, M. Fuchs: On removable singularities of $p$-harmonic maps. Analyse non linéaire, 7, No. 5 385-405 (1990)

[10] F. Duzaar, M. Fuchs: Existence and regularity of functions which minimize certain energies in homotopy classes of mappings. Asymptotic Analysis 5, 129-144 (1991)

[11] J. Eells, L. Lemaire: Another report on harmonic maps. Bull. London Math. Soc. 20, 385-524 (1988)

[12] J. Eells, J. H Sampson: Harmonic mappings of Riemannian manifolds. Am. J. Math. 86, 109-169 (1964)

[13] J. Eells, J. C. Wood: Restrictions on harmonic maps of surfaces. Topology 15, 263$266(1976)$

[14] A. Fardoun, R. Regbaoui: Équation de la chaleur pour les applications $p$ harmoniques entre variétés riemanniennes compactes. (French) C. R. Acad. Sci. Paris Sér. I Math. 333, no. 11, 979-984 (2001) 
[15] A. Fardoun, R. Regbaoui: Heat flow for $p$-harmonic map with small initial data. To appear in Calc. Var. Partial Differential Equations

[16] A. Fardoun, R. Regbaoui: Heat flow for $p$-harmonic maps between compact Riemannian manifolds. To appear in Indiana Univ. Math. J.

[17] H. Federer: Geometric measure theory. Springer, 1969

[18] M. Fuchs: Some regularity theorems for mappings which are stationary points of the $p$-energy functional. Analysis 9, 127-143 (1989)

[19] M. Fuchs: p-harmonic obstacle problems. Part III: Boundary regularity. Annali Mat. Pura Applicata 156, 159-180 (1990)

[20] R. S. Hamilton: Harmonic maps of manifolds with boundary. Lect. Notes Math. 471, Springer, Berlin, 1975

[21] R. Hardt, F.H. Lin: Mappings minimizing the $L^{p}$ norm of the gradient. Comm. Pure and appl. Math 15, 555-588 (1987)

[22] N. Hungerbühler: p-harmonic flow. Diss. Math. Wiss. ETH Zürich, Nr. 10740, 1994. Ref.: Michael Struwe; Korref.: Jürgen Moser, Zürich, 1994

[23] N. Hungerbühler: Global weak solutions of the $p$-harmonic flow into homogeneous spaces. Indiana Univ. Math. J. 45/1, 275-288 (1996)

[24] N. Hungerbühler: Compactness properties of the $p$-harmonic flow into homogeneous spaces. Nonlinear Anal. 28/5, 793-798 (1997)

[25] N. Hungerbühler: Non-uniqueness for the p-harmonic flow. Canad. Math. Bull. 40/2, 174-182 (1997)

[26] N. Hungerbühler: $m$-harmonic flow. Ann. Scuola Norm. Sup. Pisa Cl. Sci. (4) XXIV, 4, 593-631 (1997)

[27] N. Hungerbühler: A refinement of Ball's Theorem on Young measures. New York J. Math. 3, 48-53 (1997)

[28] N. Hungerbühler: Young measures and nonlinear PDEs. Habilitationsschrift ETH Zürich, 2000

[29] N. Hungerbühler: Quasilinear parabolic systems in divergence form with weak monotonicity. Duke Math. J. 107/3, 497-520 (2001)

[30] L. Lemaire: Applications harmoniques de surfaces riemannienne. J. Diff. Geom. 13, 51-78 (1978)

[31] X. G. Liu, S.H. Li: The $p$-harmonic heat flow with potential into homogeneous spaces. Acta Math. Sin. (Engl. Ser.) 18, no. 1, 21-26 (2002)

[32] X. G. Liu: A note on heat flow of $p$-harmonic mappings. (Chinese) Kexue Tongbao (Chinese) 42, no. 1, 15-18 (1997)

[33] X. G. Liu: A remark on p-harmonic heat flows. Chinese Sci. Bull. 42, no. 6, 441-444 (1997) 
[34] M. Misawa: On regularity for heat flows for $p$-harmonic maps. Proceedings of the Korea-Japan Partial Differential Equations Conference (Taejon, 1996), 14 pp., Lecture Notes Ser., 39, Seoul Nat. Univ., Seoul, 1997

[35] M. Misawa: Existence and regularity results for the gradient flow for $p$-harmonic maps. Electron. J. Differential Equations, No. 36, 17 pp. (1998)

[36] M. Misawa: Existence and regularity results for the gradient flow for $p$-harmonic maps. Regularity, blowup and related properties of solutions to nonlinear evolution equations (Japanese). (Kyoto, 1997). Sūrikaisekikenkyūsho Kōkyūroku No. 1045, 57-72 (1998)

[37] M. Misawa: On the $p$-harmonic flow into spheres in the singular case. Nonlinear Anal. 50, no. 4, Ser. A: Theory Methods, 485-494 (2002)

[38] J. Sacks, K. Uhlenbeck: The existence of minimal immersions of 2-spheres. Ann. of Math. 113, 1-24 (1981)

[39] R.S. Schoen, K. Uhlenbeck: Approximation theorems for sobolev mappings, preprint

[40] P. Strzelecki: Regularity of $p$-harmonic maps from the $p$-dimensional ball into a sphere. Manuscr. Math. 82, No.3-4, 407-415 (1994)

[41] P. Strzelecki: Stationary $p$-harmonic maps into spheres. Janeczko, Stanislaw (ed.) et al., Singularities and differential equations. Proceedings of a symposium, Warsaw, Poland. Warsaw: Polish Academy of Sciences, Inst. of Mathematics, Banach Cent. Publ. 33, 383-393 (1996)

[42] E. Zeidler: Nonlinear functional analysis and its applications,Vol. II/B: Nonlinear Monotone Operators. New York: Springer, 1990

[43] C. Q. Zhou: Compactness properties of heat flows for weakly $p$-harmonic maps. (Chinese) Acta Math. Sinica 41, no. 2, 327-336 (1998)

[44] C. Q. Zhou, Y D. Wang: Existence and nonuniqueness for the flow of $p$-harmonic maps. (Chinese) Acta Math. Sinica 41, no. 3, 511-516 (1998) 